\title{
Localization of Scatterers in Railway Environments for Train-to-Train Propagation
}

\author{
Paul Unterhuber*, Michael Walter*, Thomas Kürner ${ }^{\dagger}$ \\ ${ }^{*}$ Institute of Communications and Navigation, German Aerospace Center (DLR), Oberpfaffenhofen, 82234 Wessling, \\ Germany, paul.unterhuber@dlr.de \\ ${ }^{\dagger}$ Institute for Communications Technology, Technische Universität Braunschweig, 38106 Braunschweig, Germany
}

\begin{abstract}
Wireless communication became a key technology in the transportation domain. An increase of efficiency and safety can be achieved by connecting all traffic participants. In the railway domain wireless communication could enable new applications. In this contribution we focus on train-totrain (T2T) communication and investigate the propagation conditions. In typical railway environments different types of objects along the track highly influence the propagation channel and, in turn, the performance of wireless T2T communications. Therefore, the characterization and modeling of the propagation effects are indispensable. In comparison to cellular or vehicle-tovehicle communication, T2T propagation is hardly investigated. In this paper we present how key parameters of the multipath components (MPCs) of the channel impulse response can be estimated. The estimation is based on measurement data. We estimate delay and Doppler of single-bounce MPCs and estimate the location of the corresponding scatterers.
\end{abstract}

Index Terms - train-to-train, high speed train, propagation, measurement, scatterer location.

\section{INTRODUCTION}

The characterization of propagation effects and resulting channel modeling is essential for new communication standards. In railways, a common communication standard is Global System for Mobile Communications - Railway (GSM-R) and in future it might be Long-Term Evolution Railway (LTE-R) as discussed in [1]. Both communication standards are train-to-ground (T2G) based. A comprehensive overview of $\mathrm{T} 2 \mathrm{G}$ channel measurements and models for high speed railway (HSR) is given in [2]. In [3] future railway radio communications services and the need of a combination of $\mathrm{T} 2 \mathrm{G}$ and $\mathrm{T} 2 \mathrm{~T}$ are discussed. As a result, new applications like autonomously driving trains and virtual coupling of trains, based on ultra reliable low latency communication might be realized. The low latency requirement could be met by T2T communications. In [4] a survey on T2T communication research is given and gaps are pointed out. In comparison to T2G, T2T is hardly investigated and therefore wideband channel sounding measurements and models are missing.

In [5] a general overview of channel models is given and advantages of geometry-based stochastic channel model (GSCM) are described [6]. In fact, GSCM was introduced for multiple antenna cellular networks and single-bounce scattering by [6]. Over the last decades channel models were adapted for new applications, especially GSCM for vehicleto-vehicle propagation [7]. A first step towards a GSCM for $\mathrm{T} 2 \mathrm{~T}$ communication is the identification and characterization of scatterers. For railways typical scatterers are catenary, the signaling system and supporting masts. Furthermore, different types of artificial or natural objects could cause scattering.

Channel sounding data for railway environments was collected at an extensive T2T campaign in Italy. This campaign with two high speed trains, the environment and the measurement equipment were first described in [8]. The measurements were conducted in single-input single-output (SISO) mode with a moving transmitter (Tx) and a moving receiver (Rx). Hence, neither information on the angle of departure nor on the angle of arrival of MPCs could be derived. A first post processing step of the measured channel impulse response was done with the Kalman enhanced super resolution tracking (KEST) algorithm. Thus, MPCs have been extracted and tracked over time and the corresponding parameters like the complex amplitude, delay and life time have been derived in [9].

For the following analysis we present one example scenario of the T2T measurement in a rural environment. In a 2Dmodel we rebuilt the measurement environment of the example scenario. For simplicity reason, only catenary masts and one cross bridge are taken into account. All objects in the 2Dmodel are represented as point scatterers.

In this paper we propose to localize a scatterer through precise estimation of both delay and Doppler over the complete lifetime of the MPC. Note that we also use the accurate position, heading and velocity estimates of both trains. These estimates are obtained from a geodetic global navigation satellite system (GNSS) receiver. The Doppler is estimated with the help of the phase information for each MPC.

The paper is structured as follows: The parameter estimation is described in Section II. In Section III we present the measurement example scenario and the 2D-model. The scatterer localization is shown for both, the example scenario of the measurement and the 2D-model. The estimated delay and Doppler are shown based on measurement data and the 2D-model and differences are highlighted in Section IV. We conclude the paper in Section V. 


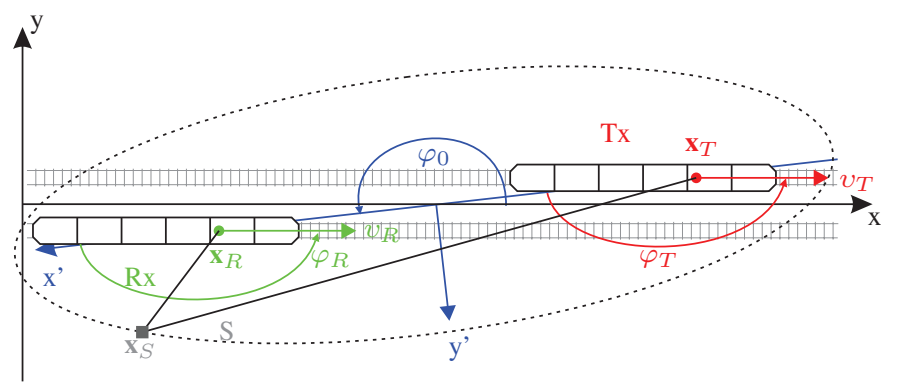

Fig. 1. One train as Tx with the position $\mathbf{x}_{T}$ marked as red dot on top right A second train as $\mathrm{Rx}$ with the position $\mathbf{x}_{R}$ marked as green dot on bottom left. One exemplary scatterer with the position $\mathbf{x}_{S}$ marked as gray square. The ellipse represents the delay of the MPC caused by the scatterer S.

\section{PARAmeter EStimation}

As mentioned before, we propose to localize a scatterer through precise estimation of both delay and Doppler over the complete lifetime of the MPC. A MPC occurs, if a wave emitted by the Tx hits a scatterer and the scattered wave is received by the Rx. If the wave is scattered only once, we call it single-bounce MPC.

The geometry between the Tx, Rx and one scatterer $\mathrm{S}$ is shown in Fig. 1. The line of sight $(\mathrm{LoS})$ path between the Tx position $\mathbf{x}_{T}$ and the Rx position $\mathbf{x}_{R}$ defines the local coordinate system with the $x^{\prime}$ - and $y^{\prime}$-axis. In the local coordinate system, the $\mathrm{Rx}$ is always in front of the Tx independent of the direction of driving without loss of generality. The global coordinate system is represented by the $x$ - and $y$-axis. The rotation angle between the local and the global coordinate system is $\varphi_{0}$. The heading of the Tx is described by $\varphi_{T}$ and the heading of the $\mathrm{Rx}$ by $\varphi_{R}$. The scatterer $\mathrm{S}$ is static and represented as gray square with the position $\mathbf{x}_{S}$.

\section{A. Representation of the delay}

Each scatterer causes a certain MPC with a delay $\tau_{i}(t)$ at time $t$. This delay is represented by an ellipse in Fig. 1. The ellipse is defined with their foci at Tx and Rx as

$$
a_{\tau_{i}(t)}=\frac{\tau_{i}(t) \cdot c}{2}
$$

and

$$
b_{\tau_{i}(t)}=\sqrt{\left(\frac{\tau_{i}(t) \cdot c}{2}\right)^{2}-\left(\frac{\left\|\mathbf{x}_{T}(t)-\mathbf{x}_{R}(t)\right\|}{2}\right)^{2}}
$$

where $a_{\tau_{i}(t)}$ represents the semi-major axis and $b_{\tau_{i}(t)}$ the semiminor axis [10]. Every scatterer position $\mathbf{x}_{S}^{\prime}$ on the ellipse can be calculated by

$$
\mathbf{x}_{S}^{\prime}\left(t ; \phi \mid \tau_{i}(t)\right)=\left[\begin{array}{l}
\dot{a} \\
\dot{b}
\end{array}\right]=\left[\begin{array}{ll}
a_{\tau_{i}(t)} & \cos (\phi) \\
b_{\tau_{i}(t)} & \sin (\phi)
\end{array}\right] .
$$

The angle $\phi \in(-\pi, \pi]$ determines the position of the scatterer on the ellipse, but is not the polar angle between the scatterer and the $\mathrm{x}$-axis [11].

\section{B. Representation of the Doppler}

In $\mathrm{T} 2 \mathrm{~T}$ or vehicle-to-vehicle communication $\mathrm{Tx}$ and $\mathrm{Rx}$ are moving. Therefore, the transmitted signal experiences a Doppler $f_{d}$ [11]. A common representation of the Doppler is based on the velocities of Tx and Rx, and the angle of departure and angle of arrival. Due to the SISO measurements, neither angle of departure nor angle of arrival are directly available. Alternatively, the Doppler can be expressed by the delay changes as shown in (4), with the delay $\tau_{i}\left(\mathbf{x}_{S}, t\right)$ with respect to the scatterer position vector $\mathbf{x}_{S}$ and time $t$ times the frequency $f_{c}[11]$.

$$
f_{d}\left(\mathbf{x}_{S}, t\right)=-\frac{\partial}{\partial t} \tau_{i}\left(\mathbf{x}_{S}, t\right) f_{c} .
$$

Instead of the delay, the phase information can be used for the Doppler estimation as

$$
f_{d}\left(\mathbf{x}_{S}, t\right)=-\frac{\partial}{\partial t} \theta_{i}\left(t, \tau_{i}\left(\mathbf{x}_{S}, t\right)\right) \frac{1}{2 \pi \cdot \Delta t}
$$

where $\Delta t$ is the time between two consecutive measurements.

Following the derivation of the Doppler [11] and adopting the parameters to the T2T scenario as shown in Fig. 1, the Doppler related to every position $\mathbf{x}_{S}^{\prime}$ on the ellipse can be calculated as follows:

$$
\begin{aligned}
& f_{d}\left(\mathbf{x}_{S}^{\prime}\left(t ; \phi \mid \tau_{i}(t)\right)\right)= \\
& \left(\frac{\dot{a}+d(t) / 2}{\sqrt{(\dot{a}+d(t) / 2)^{2}+\dot{b}^{2}}} v_{T} \cos \left(\varphi_{T}(t)\right)\right. \\
& +\frac{\dot{a}-d(t) / 2}{\sqrt{(\dot{a}-d(t) / 2)^{2}+b^{2}}} v_{R} \cos \left(\varphi_{R}(t)\right) \\
& +\frac{\dot{b}}{\sqrt{(\dot{a}+d(t) / 2)^{2}+b^{2}}} v_{T} \sin \left(\varphi_{T}(t)\right) \\
& \left.+\frac{\dot{b}}{\sqrt{(\dot{a}-d(t) / 2)^{2}+\dot{b}^{2}}} v_{R} \sin \left(\varphi_{R}(t)\right)\right) \frac{f_{c}}{c} .
\end{aligned}
$$

The variable $d$ represents the LoS distance, $v_{T}$ the speed of the $\mathrm{Tx}$ and $v_{R}$ the speed of the $\mathrm{Rx}$.

\section{MeAsurement EXAMPLE SCEnARIO AND 2D-MODEL}

\section{A. Measurement example scenario}

The T2T measurement campaign was performed in typical railway environments with the DLR RUSK channel sounder at $f_{c}=5.2 \mathrm{GHz}$ and with a bandwidth of $120 \mathrm{MHz}$ as presented in [8]. A first analysis of the processed data with the KEST algorithm showed very pronounced MPCs in rural environments [9]. Therefore, a rural environment was chosen as example scenario. A $t=20 \mathrm{~s}$ long sequence of the measurement data was used for the following analysis. In this sequence, the Tx was driving with a velocity $v_{T}=50 \mathrm{~km} / \mathrm{h}$ in front of Rx with a velocity $v_{R}=10 \mathrm{~km} / \mathrm{h}$; the distance between $\mathrm{Tx}$ and $\mathrm{Rx}$ increased from $d=540 \mathrm{~m}$ to $760 \mathrm{~m}$. This position and velocity data was obtained by geodetic GNSS receivers and is used 
TABLE I

POSITION AND VELOCITY OF ALL OBJECTS IN THE 2D-MODEL

\begin{tabular}{|c|c|c|c|c|}
\hline Object & \multicolumn{2}{|c|}{ Position } & \multicolumn{2}{c|}{ Velocity } \\
& $\mathrm{x}$ & $\mathrm{y}$ & \multicolumn{2}{c|}{$v$} \\
& {$[\mathrm{~m}]$} & {$[\mathrm{m}]$} & {$[\mathrm{km} / \mathrm{h}]$} & {$[\mathrm{m} / \mathrm{s}]$} \\
\hline $\mathrm{Tx}$ & 0 & 3.25 & 50 & 13.89 \\
$\mathrm{Rx}$ & -545 & -3.25 & 10 & 2.78 \\
Scatterers & $14,70,125$, & 8 & 0 & 0 \\
& $179,181,238$ & & & \\
& $295,332,375$ & & & \\
\hline
\end{tabular}

as ground truth. Due to the rural environment and the roof mounted GNSS antennas, we assume open sky condition.

The scatterer localization algorithm for the example scenario consists of several steps:

- Step one: The recorded GNSS data is used to estimate the positions and velocities of Tx and Rx. Furthermore, the geodetic WGS 84 datum is transformed into a datum in a Cartesian coordinate system. The distance $d$ between Tx and Rx is calculated.

- Step two: The complex amplitude, delay and life time of each MPC is estimated by the KEST algorithm [9].

- Step three: the Doppler $f_{d}\left(\mathbf{x}_{S}^{\prime}\left(t ; \phi \mid \tau_{i}(t)\right)\right)$ of all scatterers which induce the same delay $\tau_{i}(t)$ are estimated from (6).

- Step four: The phase information $\theta_{i}$ of the estimated complex amplitude is used to compute the $f_{d}\left(\mathbf{x}_{S}, t\right)$ from (5). Note that $\Delta t=1.024 \mathrm{~ms}$.

- Step five: $f_{d}\left(\mathbf{x}_{S}, t\right)$ is compared with $f_{d}\left(\mathbf{x}_{S}^{\prime}\left(t ; \phi \mid \tau_{i}(t)\right)\right)$. The result of this comparison is a pair of positions or several pairs for one scatterer.

- Last step: The positions of the Tx and $\mathrm{Rx}$ and the estimated scatterer positions are overlaid onto a georeferenced satellite image. Hence, the scatterer can be identified in the satellite image.

\section{B. 2D-model}

We introduce an artificial scenario based on the geometry of the measurement example scenario in a 2D-model. The 2D-model uses a Cartesian coordinate system and contains the track layout, the moving $\mathrm{Tx}$ and $\mathrm{Rx}$ and all potential scatterers whose positions have been estimated based on the measurement data. These scatterers are modeled by perfect point scatterers. The velocities and start positions of Tx and $\mathrm{Rx}$ and the positions of the static scatterers are listed in Table I. A modified scatterer localization algorithm is used:

- Step one: The velocities and start positions of Tx and Rx are known. The distance $d$ is calculated.

- Step two: A ray tracing is performed to estimate the delay $\tau_{i}(t)$ for all MPCs over a duration of 20 seconds.

- Step three is the same as above.

- Step four: The delay $\tau_{i}(t)$ is used to compute the $f_{d}\left(\mathbf{x}_{S}, t\right)$ with $f_{c}=5.2 \mathrm{GHz}$.

- Step five is the same as above.

- Last step: The positions of the Tx and Rx and the estimated scatterer positions are plotted together with the objects of the 2D model.



Fig. 2. Delay over time for the estimated MPCs. The dashed red line with the lowest delay represents the distance between Tx and Rx.

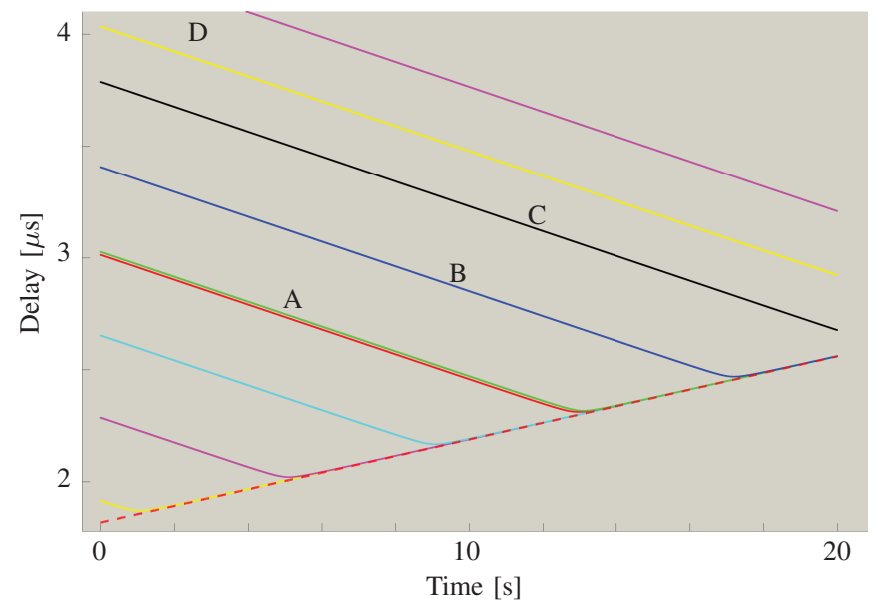

Fig. 3. Delay over time for the estimated MPCs of the 2D-model. The dashed red line with the lowest delay represents the distance between Tx and Rx.

\section{RESULTS AND COMPARISON}

The results of the delay and Doppler estimation and scatterer localization are shown in the following figures. The estimation results based on the measurement example scenario are compared with the estimation results based on the 2D-model. Four pronounced MPCs are highlighted and the corresponding scatterers identified. In Fig. 2, 4 and 6 the results of the measurement example scenario are shown; in all three figures each color represents a particular MPC. The results of the 2Dmodel are shown in Fig. 3, 5 and 7; same colors represent the same MPCs.

\section{A. Comparison of the delay behavior}

In Fig. 2 and 3 the delay is plotted over time. The distance between Tx and Rx is plotted as a reference with a solid red line.

Fig. 2 shows the parameters of only those MPCs which are continuously tracked over several seconds. The figure reveals a pattern with parallel lines where each line can be associated 


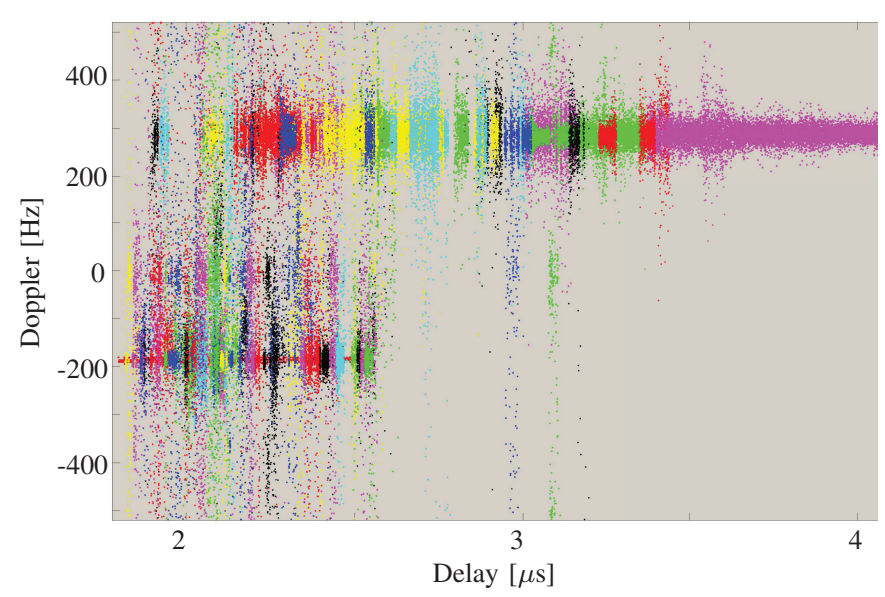

Fig. 4. Doppler over delay for the estimated MPCs.

with a MPC. We strongly assume that these MPCs are caused by scatterers. Some lines consist of segments of different color. Note that this change in color happens, if the algorithm lost track of one MPC and generates a new path with a new color of the same MPC. Four MPCs are marked with A, B, C and D. We also comment on two effects shown in Fig. 2. First, in case of A two very closely arriving MPCs can be observed between $t=9 \mathrm{~s}$ to $10 \mathrm{~s}$. The estimation before $t=9 \mathrm{~s}$ of a single MPC splits into two MPCs because of two close scatterers. Second, the MPC D is tracked over $20 \mathrm{~s}$ which leads to the assumption of very strong scattering.

In comparison, Fig. 3 shows the delay behavior of perfect point scatterers. The delay estimations based on the 2D-model show the same trends as the estimations of the measurement example scenario.

\section{B. Comparison of the Doppler behavior}

The resulting Doppler is shown in Fig. 4 and 5. Two regions can be separated. First, the Doppler values around $f_{d}=288 \mathrm{~Hz}$ are caused by scatterers placed in front of Tx and Rx. For MPCs with a delay of less than $2.6 \mu$ s the Tx passes by the scatterer within the measurement time. Therefore, the Doppler changes to the second region around $f_{d}=-192 \mathrm{~Hz}$. The mentioned Doppler behavior is clearly visible in Fig. 5, and can be assumed for Fig. 4 as well. The high Doppler spread of the measurement data might be caused by the vibrations and the relative movement of the trains.

\section{Comparison of the scatterer localization}

In Fig. 6 the position estimation is plotted onto a georeferenced satellite image. Only the area covering the four examples is plotted. The driving direction is from bottom right to top left; the green stars along the left track represent the location of the Tx. The dot and ring markers of the same color represent the estimated positions of a particular MPC. The MPCs A, B and C can be clearly identified as masts of the catenary. In case of A, the catenary was supported by two masts on the left side. Therefore, two MPCs were tracked as

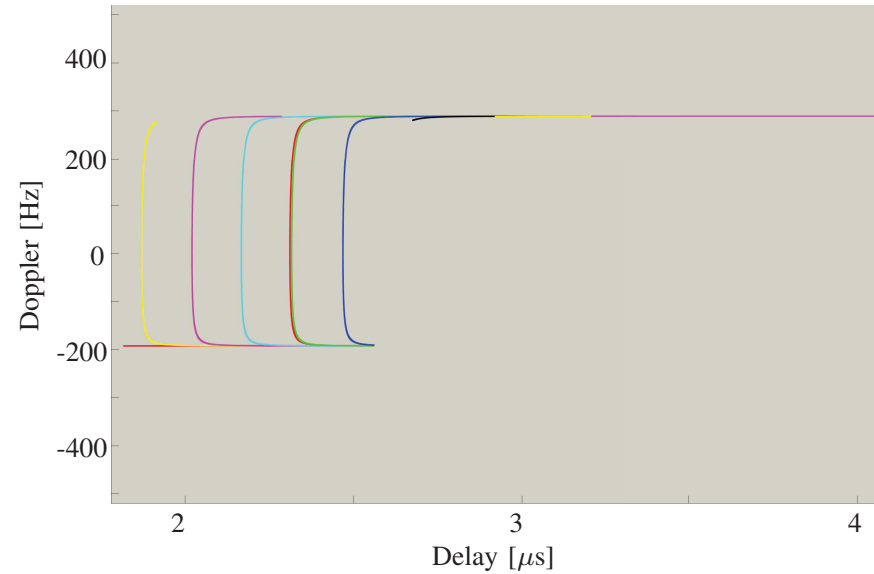

Fig. 5. Doppler over delay for the estimated MPCs of the 2D-model.

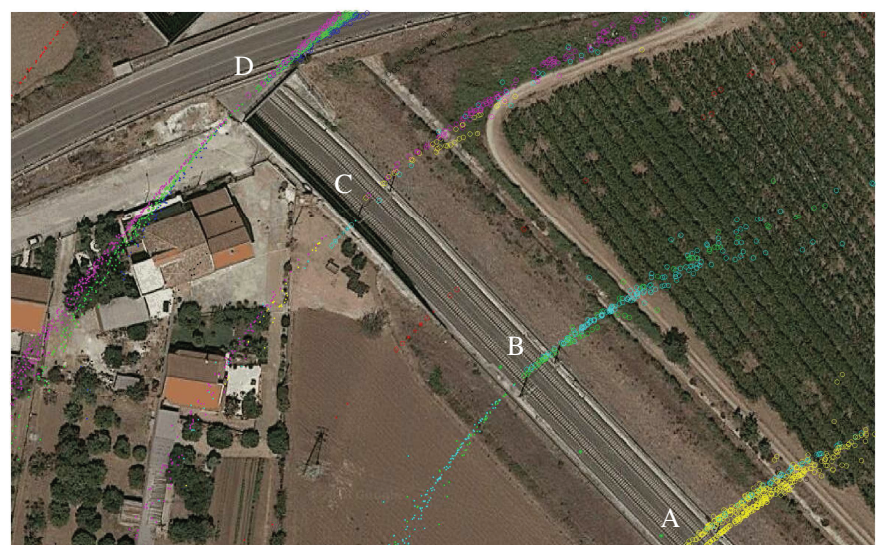

Fig. 6. Estimated scatterer position for four MPCs (A, B, C, D) plotted on Google maps. Image by Google, Map Data 2017 NOAA, Digital Globe 2018

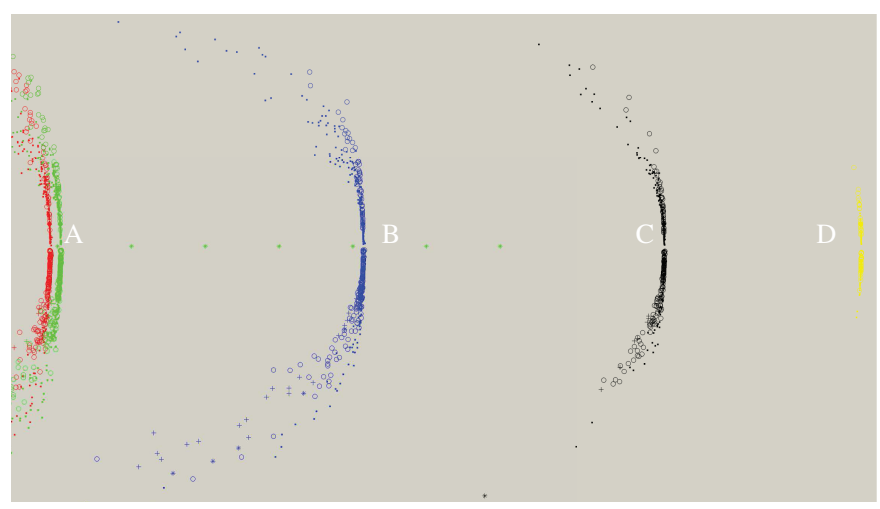

Fig. 7. Estimated scatterer position for four MPCs (A, B, C, D) of the 2Dmodel.

mentioned before. The cross bridge in the upper left corner of Fig. 6 causes the strong MPC D.

Fig. 7 holds for the 2D-model and also treats A,B,C, and D as Fig. 6. In Fig. 7 the driving direction is from left to right; the green stars represent the Tx. The position estimations reveal a similar spreading for both the measurement example scenario 


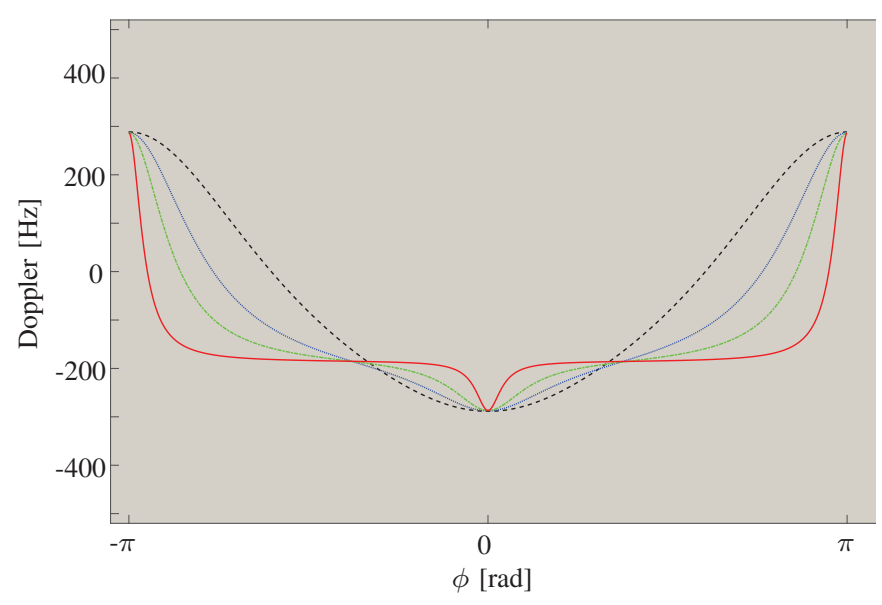

Fig. 8. Doppler over angle $\phi$ for different delays $\tau_{i}$.

and the 2D-model.

In general, position estimations of consecutive measurements of one MPC spread to one side and the corresponding ambiguity to the other side of the scatterer. The spreading of the estimated positions is caused by the comparison of the Doppler calculated by (5) for the measurements or (4) for the 2D-model with all Doppler values of possible scatterer positions along the ellipse by (6). The rotation angle $\phi$ dependent Doppler of four different delays is shown in Fig. 8. The red continuous line can be seen as worst case and the black dashed line as best case for estimating $\phi$. For example, a MPC with a short delay, would be described by the Doppler of the red line. In this case, a Doppler change by $10 \mathrm{~Hz}$ between two consecutive measurements would result in either an angular change by $85 \mathrm{deg}$ or by $0.3 \mathrm{deg}$ depending on the location of the scatterer along the ellipse. This behavior leads to a wide spread of the position estimation. The black dashed line represents a MPC with a long delay. The angular changes are more constant for similar Doppler changes. As a result, the spreading of the position estimation is reduced as for example for case D in Fig. 7.

\section{CONCLUSION}

In this contribution, we presented the localization of scatterers in railway environments. The estimated parameters are based on a T2T channel sounding measurement campaign. Although the measurements were performed in SISO mode, scatterers could be localized due to precise delay and Doppler estimation.

MPCs could be separated and tracked up to $11 \mathrm{~s}$ for the measurement example scenario. Furthermore, clear trends of MPCs could be shown in the delay and Doppler domain. Four MPC examples were highlighted and the positions of the corresponding scatterers estimated. Catenary masts and a cross bridges along the railway track could be clearly related to the MPCs in the measurement example scenario.

In addition to the measurement example scenario, the measurement environment was rebuilt in a $2 \mathrm{D}$-model based on point scatterers. A comparison between the estimation results based on the measurement example scenario and the 2Dmodel was shown. The delay behavior showed similar trends for scatterers ahead of $\mathrm{Tx}$ and $\mathrm{Rx}$ for both the measurement example scenario and the 2D-model data. Due to the large Doppler spread of the measurement example scenario, only trends of large regions of the estimated Doppler could be compared to the 2D-model based estimation. The performance of the scatterer localization algorithm was compared as well. The spreading of the position estimation for the measurement data as well as for the 2D-model data was explained. This spreading is caused by the Doppler estimation.

\section{REFERENCES}

[1] R. He, B. Ai, G. Wang, K. Guan, Z. Zhong, A. F. Molisch, C. BrisoRodriguez, and C. P. Oestges, "High-Speed Railway Communications: From GSM-R to LTE-R," IEEE Vehicular Technology Magazine, vol. 11, no. 3, pp. 49-58, Sept 2016.

[2] C. X. Wang, A. Ghazal, B. Ai, Y. Liu, and P. Fan, "Channel Measurements and Models for High-Speed Train Communication Systems: A Survey," IEEE Communications Surveys Tutorials, vol. 18, no. 2, pp. 974-987, Secondquarter 2016.

[3] J. Moreno, J. M. Riera, L. d. Haro, and C. Rodriguez, "A Survey on Future Railway Radio Communications Services: Challenges and Opportunities," IEEE Communications Magazine, vol. 53, no. 10, pp. 62-68, Oct 2015.

[4] P. Unterhuber et al., "A Survey of Channel Measurements and Models for Current and Future Railway Communication Systems," Mobile Information Systems, vol. 2016, pp. 1-14, Jun 2016. [Online]. Available: http://dx.doi.org/10.1155/2016/7308604

[5] P. Almers, E. Bonek, A. Burr, N. Czink, M. Debbah, V. Degli-Esposti, H. Hofstetter, P. Kyösti, D. Laurenson, G. Matz, A. Molisch, C. Oestges, and H. Özcelik, "Survey of Channel and Radio Propagation Models for Wireless MIMO Systems," EURASIP Journal on Wireless Communications and Networking, vol. 2007, no. 1, p. 019070, Feb 2007.

[6] A. F. Molisch, A. Kuchar, J. Laurila, K. Hugl, and R. Schmalenberger, "Geometry-Based Directional Model for Mobile Radio Channels Principles and Implementation," European Transactions on Telecommunications, vol. 14, no. 4, pp. 351-359, Jul 2003.

[7] D. W. Matolak, "Modeling the Vehicle-to-Vehicle Propagation Channel: A Review," Radio Science, vol. 49, no. 9, pp. 721-736, Sept 2014

[8] P. Unterhuber, S. Sand, M. Soliman, B. Siebler, A. Lehner, T. Strang, M. d'Atri, F. Tavano, and D. Gera, "Wide Band Propagation in Trainto-Train Scenarios Measurement Campaign and First Results," in 2017 11th European Conference on Antennas and Propagation (EuCAP), Mar 2017, pp. 3356-3360.

[9] P. Unterhuber, T. Jost, W. Wang, and T. Kürner, "Measurement Based Spatial Characteristics of MPCs in Train-to-Train Propagation," in 2018 12th European Conference on Antennas and Propagation (EuCAP), Apr 2018.

[10] I. Bronshtein, K. Semendyayev, G. Musiol, and H. Mhlig, "Handbook of Mathematics", 5th ed. Springer-Verlag Berlin Heidelberg, 2007.

[11] M. Walter, D. Shutin, and U. C. Fiebig, "Delay-Dependent Doppler Probability Density Functions for Vehicle-to-Vehicle Scatter Channels," IEEE Transactions on Antennas and Propagation, vol. 62, no. 4, pp. 2238-2249, Apr 2014. 